\title{
Umbilical Cord Cells as a Source of Cardiovascular Tissue Engineering
}

\author{
Christian Breymann, MD, *,I Dörthe Schmidt, MD, ${ }^{2}$ and S. P. Hoerstrup, MD, PhD ${ }^{2}$ \\ 'Feto-Maternal Haematology Research Group, Obstetric Research, University Hospital Zurich and GGS \\ (Obstetrics and Gynaecology Center Seefeld/Hirslanden Clinic, Zurich); and 2Department of Surgical Research \\ and Clinic for Cardiovascular Surgery, University Hospital Zurich
}

\author{
${ }^{*}$ Correspondence and reprint \\ requests to: \\ Christian Breymann, MD, \\ Obstetric Research, Feto Maternal \\ Haematology Unit, \\ Frauenklinikstr 10, \\ CH 8091 Zurich. \\ E-mail: Christian.breymann@usz.ch
}

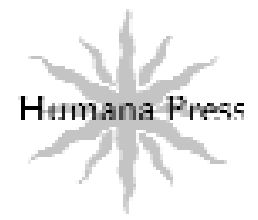

\begin{abstract}
There is increasing scientific evidence that human umbilical cord cells are a valuable source of adult stem cells that can be used for various implications including regenerative medicine and tissue engineering. The review describes the role of progenitor cells (mesenchymal, endothelial, prenatal) for the use in cardiovascular tissue engineering, i.e., the formation of large vessels and heart valves from umbilical cord cells.

Currently used replacements in cardiovascular surgery are made of foreign materials with well known drawbacks such as thrombo-embolic complications, infection, loss of functional and biological properties, and others. Especially in the field of replacements in congenital cardiac defects, there would be a need of materials which have the advantage of optimal biological and mechanical properties. In the case of human umbilical cord cells, autologous cells can be used by minimally invasive procedures. The cells have excellent growth capacities and form a neo-matrix with excellent mechanical properties. For optimal growth and modeling, scaffolds are required with high biocompatibility and biodegradability, which allow cell attachment, ingrowth, and organization. Nutrients and waste must be easily transported and cells should be in entire contact with host's body. Finally, regenerated materials can be fully incorporated and the scaffold is completely replaced. Besides these cell and scaffold requirements, feto-maternal conditions and risk factors concerning deriving stem cells are of major interest. There are still many open questions concerning whether and how maternal conditions such as infection (viral or bacterial) or gestational age of the newborn influence stem cell harvesting and quality. If these cells will be used for the construction of replacement materials, it is clear that very strict criteria and protocols be introduced enabling the promising step from isolated cells to a therapeutic device such as a new heart valve. It is hoped that it will be only a question of time until human umbilical cord cells will be used frequently as the source of cardiovascular tissues among others in the clinical setting of treating congenital heart defects.
\end{abstract}

Index Entries: Human umbilical cord; stem cells; tissue engineering; cardiovascular; heart defect; congenital.

\section{Introduction}

To date, it is well known and evident that adult stem cells, also named progenitor cells, play an important role in regenerative medicine and even enable the mechanisms of tissue regeneration. After tissue damage or destruction in an in vivo environment, they can be recruited from several sources for example, bone marrow, blood, or as resident stem cell from the injured tissue itself in order to start the cascade for tissue repair. 
More than 30,000 articles have been published on stem cell research demonstrating the increased interest in this field. The dynamics of stem cell research have been driven particularly by tissue engineering as a major discipline in regenerative medicine with focus on various organs such as skin, bone and cartilage tissue, urinary tract system, liver parenchyma, eyes, neuronal tissue, and finally the cardiovascular system. Furthermore, there is an increasing interest among the public and the mass media and it seems only to be a matter of time until tissue engineering reaches the routine medical practice (1).

In this review, a short summary regarding to the role of progenitor cells derived from umbilical cord for cardiovascular tissue engineering is presented. The umbilical cord provides various types of progenitor cells including umbilical cordderived mesenchymal cells, endothelial progenitor cells (EPC), and cells from the Wharton's jelly tissue (2). Thus, using human umbilical cord (hUC)-derived cells could be an attractive concept for regenerative medicine. This will be highlighted in another article in this issue by $\mathrm{C}$. Cetrulo.

\section{The Potential Role of Umbilical Cord-Derived Cells in Cardiovascular Tissue Engineering}

Cardiovascular tissue engineering, focuses on the in vitro fabrication of autologous, living tissues. This has potential for regeneration and is a promising scientific field, addressing the so far unmet medical need for growing replacements, particularly for the repair of congenital malformation.

Currently used replacements are nonliving and based on foreign materials. They exhibit lack of growth and remodeling, and also carry the risks for thrombo-embolic complications and infections. Particularly, in today's congenital heart surgery, there is a substantial need for appropriate, growing replacement materials for the repair of congenital cardiac defects. The surgical treatment is commonly based on nonautologous valves or conduits $(3,4)$ with disadvantages including obstructive tissue ingrowths and calcification of the replacement. These limitations and the lack of growth typically necessitate various reoperations of the pediatric patients with cardiovascular defects and each time they are associated with increased morbidity and mortality.

Tissue-engineered constructs based on umbilical cordderived cells could be an attractive alternative to the current replacement materials for the repair of congenital malformations. Being living material and owing to their progenitor characteristics having the capacity of self-remodeling. Here, umbilical cord-derived cells would be ideal for tissueengineered constructs as they can be harvested without harming intact donor structures and high risks for the child. The advantages of using umbilical cord-derived cells are summarized in Table 1.

\section{The Concept of In Vitro Generating of Umbilical Cord-Derived Cell Based Cardiovascular Replacements}

Tissue engineering using umbilical cord-derived cells could enable living autologous replacement structures including vascular grafts and heart valves. Therefore, cells could be harvested from the umbilical cord, differentiated and expanded in vitro.
Table 1

Advantages of Using hUC-Derived Cells for Cardiovascular Tissue Engineering

- Autologous cells source

- Minimally invasive obtainable

- Cell source providing different types of cells including progenitor cells

- Excellent cell growth capacities

- Forming an optimal neo-matrix with excellent mechanical properties

In order to fabricate the cardiovascular constructs, cells should be seeded onto biodegradable scaffold that are implanted into a biomimetic system (bioreactor). The tissue formation and maturation would have been accelerated (5). After $14 \mathrm{~d}$, sufficient tissue formation should have been taken place and the constructs should be ready for implantation.

In order to create a functional heart valve with the mechanical properties of the native counterpart, rapid development of the extracellular matrix is crucial. Therefore, the choice of cells, which are responsible for the production of extracellular matrix is an important factor. Two cell types are routinely used for the fabrication of cardiovascular tissues: cells with the capacity to form extracellular matrix (commonly myofibroblasts) and endothelial cells with antithrombogenic characteristics. The seeding procedure is mostly performed sequentially onto three-dimensional scaffolds: first, by seeding of the myofibroblasts and then followed by the endothelial cells (6). From umbilical cord, myofibroblast could be obtained from its vessels or Wharton's jelly. Endothelial cells could be isolated either from the vessels or alternatively from the umbilical cord blood.

As a temporary vehicle for cells, biodegradable scaffolds can be used in cardiovascular tissue engineering. Possible problems concerning these systems are systemic toxicity, growth limitation, differentiation and function restraints, incorporation barriers, and cell or tissue delivery difficulties.

The ideal scaffold would have a high porosity and interconnected pores with pore size between 100 and $150 \mu \mathrm{m}$ enabling cell differentiation and ingrowth, diffusion of nutrients and waste as well as the contact between cells and the host's body. The scaffold material should be biodegradable. Mechanical strength with controlled degradation is necessary, which enables elastic properties up to strains of $10 \%$. Numerous scaffolds have been used and tested such as: PGA, PLA, P3HB, P4HB, Degrapol, PGA + P4HB, PGA + Degrapol, and PGA + PCL). Scaffold requirements are listed in Table 2.

In general the concept of cardiovascular tissue engineering has been already validated in large animal studies (7). Completely autologous, living trileaflet heart valve has been successfully implanted in a growing sheep model for up to $20 \mathrm{wk}$. These valves showed good functional performance as well as structural and biomechanical characteristics strongly resembling those of native semilunar heart valves. The first clinical application of a tissue-engineered pulmonary artery graft in a pediatric patient was reported by Shinoka et al (8). However, long-term results have not been presented so far. 
Table 2

Requirements of an Ideal Scaffold

- Biocompatibility

- Biodegradability

- Allowing cell attachment, ingrowth, and cell organization

- Diffusion of nutrients and waste

- Cells in contact with host's body

- Full incorporation and replacement

Table 3

Obstetric Patients and Procedure for Umbilical Cord Sampling

- Elective caesarean section.

- Term birth (>37 WOG)

- Normal feto-maternal history

- Normale bacterial and serological testing

- No amnion rupture, no sign of amnion infection

- Umbilical cord and cord blood sampling in $5 \mathrm{~min}$ period after clamping

$10 \mathrm{~mL}$ Hank solution with ethylenediamine tetra-acetic acid

Medium: DMEM (Dulbecco's medium)

Table 4

Steps in Tissue Engineering From hUCC

- Cell isolation and cultivation

- Bioabsorbable scaffold (4-6 wk)

- Cell seeding, in vitro culture

- Pulse duplicator culture environment

"Bioreactor"

Accelerates tissue formation and maturation

\section{Summary of Own Research in the Field}

Since 2001, human umbilical cord cells (hUCC) were investigated in a cooperation project as a source of cardiovascular tissue engineering. Umbilical cord tissues and blood were sampled in the obstetric department according to a fixed protocol for the obstetric patients undergoing elective caesarean delivery (Table 3).

After cell isolation and cultivation, cells are seeded on the bioabsorbable scaffold for 4-6 wk and in vitro cultured in a pulse duplicator culture environment which mimics circulatory characteristics (bioreactor) and therefore accelerates tissue formation and maturation (Table 4). The analysis of the cells and derived tissue-engineered implants are listed in Table 5.

\section{Chronological Course of Studies}

In 2002, the feasibility of using umbilical cord cells (UCC) as an alternative autologous cell source for cardiovascular tissue engineering was investigated. The feasibility of creating pulmonary artery conduits from hUCC was investigated $(9,10)$.

\section{Methods}

The hUCCs were harvested and expanded in culture. Pulmonary conduits fabricated from rapidly bioabsorbable polymers were seeded with hUCC and grown in vitro in a pulse duplicator bioreactor.
Table 5

Analysis of Tissue-Engineered Structures

- Flow cytometry

ASMA

Vimentin

- Histology, immunohistochemistry Collagen, AMSA, vimentin

- Micro- and ultrastructure

- Quantitative analysis Comparison with native tissue

- Biomechanical analysis Mechanical properties

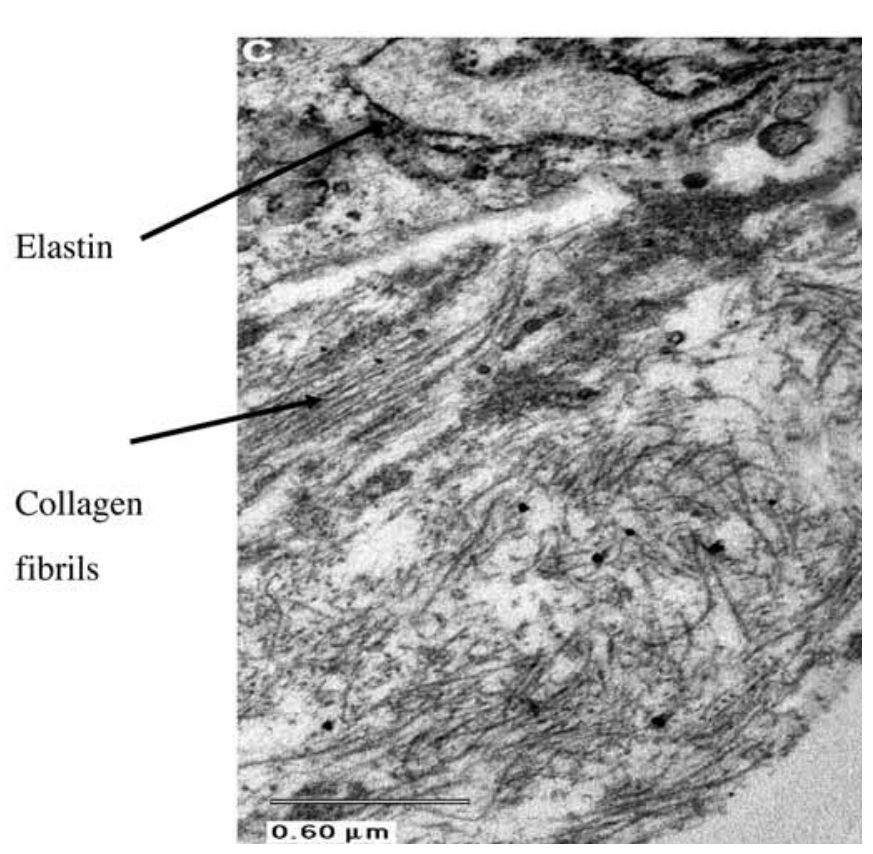

Fig. I. Flow cytometry of human saphenous vein-derived cells (upper panel) and hUC-derived cells demonstrates a similar staining pattern for the expression of vimentin and $\alpha$-smooth muscle actin.

Morphological characterization of the generated neo-tissues included histology, transmission, and scanning electron microscopy. Characterization of extracellular matrix was performed by immunohistochemistry. Extracellular matrix protein content and cell proliferation were quantified by biochemical assays. Biomechanical testing was performed using stress-strain and burst-stress tests.

\section{Results}

Histology of the conduits revealed viable, layered tissue, and extracellular matrix formation with glycosaminoglycans and collagens I and III. Cells stained positive for vimentin and $\alpha$-smooth muscle actin (ASMA). Scanning electron microscopy showed confluent homogenous tissue surfaces. Transmission electronmicroscopy demonstrated elements typical of viable myofibroblasts, such as collagen, fibrils, and elastin (Fig. 1). Extracellular matrix proteins were significantly lower compared with native tissue and increase in the cell content was increased. The mechanical strength of the pulsed constructs was comparable with native tissue; the static controls were significantly weaker. 


\section{Conclusions}

In vitro fabrication of tissue-engineered human pulmonary conduits was feasible utilizing hUCC and a biomimetic culture environment. Morphological and mechanical features approximated human pulmonary artery. The hUCC demonstrated excellent growth properties representing a new, readily available cell source for tissue engineering without necessitating the sacrifice of intact vascular donor structures.

In 2004 the results from testing of various umbilical tissues as sources for tissue engineering were published (11). This study evaluated cells isolated from human umbilical cord artery (UCA), umbilical cord vein (UCV), and whole cord as alternative autologous cell sources for cardiovascular tissue engineering.

\section{Methods}

Cells were isolated from UCA, UCV, UCC, and saphenous vein segments (VC) from adults, and were expanded in culture. All three expanded cell groups were seeded on bioabsorbable copolymer strips and were grown in vitro for $28 \mathrm{~d}$. Isolated cells were characterized by flow cytometry, histology, immunohistochemistry, proliferation assays and compared to VC. Morphological analysis of the seeded polymer strips included histology, immunohistochemistry, sodium dodecyl sulfate-polyacrylamide gel electrophoresis, transmission electron microscopy, scanning electron microscopy, and uniaxial stress testing.

\section{Results}

UCA, UCV, and UCC demonstrated excellent cell growth properties comparable to VC. Following isolation, all three cell groups showed myofibroblast-like morphology and characteristics by staining positive for ASMA and vimentin. Histology and immunohistochemistry of seeded polymers showed good tissue and extracellular matrix formation containing collagen I, collagen III, and elastin. Transmission electron microscopy showed viable myofibroblasts and the deposition of collagen fibrils and progessive growing tissue formation, with a confluent surface, was observed in scanning electron microscopy. No difference was found among the mechanical properties of UCA, UCV, UCC, and VC tissue-engineered constructs.

\section{Conclusions}

Tissue engineering of cardiovascular constructs by using UCA, UCV, and UCC is feasible in an in vitro environment. Cell growth, morphology, characteristics and tissue formation were comparable between UCA, UCV, UCC, and VC. UCC represent an attractive, readily available autologous cell source for cardiovascular, tissue engineering offering the additional benefits of utilizing juvenile cells and avoiding the invasive harvesting of intact vascular structures.

As a next step, it was interesting to investigate EPC from umbilical cord blood as a source for cardiovascular tissue engineering (12). Here, tissue-engineered vascular grafts using hUC blood-derived EPCs, as a prenatal available cell source for pediatric applications were focussed.

\section{Methods}

EPCs were isolated from $20 \mathrm{~mL}$ fresh hUC blood by gradient centrifugation and cultured in endothelial basal medium containing growth factors.
After proliferation and differentiation cells were analyzed by immunohistochemistry and seeded onto three-dimensional biodegradable vascular scaffolds (porosity >95\%, $n=22$ ). Twenty-four hours after seeding, the vascular grafts were positioned into a pulse-duplicator in vitro system and grown for 48 h under biomimetic conditions. Asecond group was grown $6 \mathrm{~d}$ statically and an additional $6 \mathrm{~d}$ biomimetically. Controls were cultured statically. Analysis of the grafts included immunohistochemistry, histology, and scanning electron microscopy.

\section{Results}

Preseeding differentiated EPCs demonstrated constant endothelial phenotypes and showed typical endothelial characteristics including uptake of acetylated low-density lipoprotein, cluster of differentiation 31, von Willebrand factor, and endothelial nitric oxide synthetase. Seeded EPCs revealed favorable cell-to-polymer attachment and proliferation into the 3D tubular scaffolds. Both conditioned and static cellular constructs demonstrated positive staining for cluster of differentiation 31, von Willebrand factor, and expression of endothelial nitric oxide synthase.

\section{Conclusions}

HUC-derived EPCs demonstrated exceptional growth characteristics. These cells demonstrated a constant endothelial phenotype and related functional features. Based on these results EPCs seem to be a promising autologous cell source regarding cardiovascular tissue engineering, particularly for the repair of congenital defects.

In the following study, it was recently shown that the endothelial tissues derived from EPC can be seeded on myofibroblasts derived from umbilical cord fibroblasts and finally form living patches, which can be used as replacements (13).

\section{Future Perspective-Prenatal Harvested Cells}

Of particularly importance for the pediatric cardiovascular tissue engineering is cell-harvesting at early stage that allows having the tissue-engineered replacement ready for implantation at the birth of the patient. Therefore, cells should be harvested prenatally at an early stage of pregnancy without harming any intact childish donor structures. As used in routinely prenatal diagnostics, both the amniotic fluid and the placenta could provide the least invasive access to fetal cells (1). Few studies describe prenatal ewe's cells from amniotic fluid as a new cell source for tissue engineering for diaphragma reconstruction in an animal model. Kaviani et al. reported the use amniotic fluidderived cells obtained at 16-21 wk compared with postnatal human placental cells obtained from cesarean section delivered placenta at 33-35 wk of gestation. Recently engineering of living autologous pediatric cardiovascular tissue replacements based on prenatal progenitor cells were investigated (14).

\section{Conclusion}

In conclusion hUCC are a promising cell source for cardiovascular tissue engineering since they have demonstrated excellent growth properties and phenotypes similar to the native counterparts of the tissue they should replace. The HUC is an excellent and unique source of various types of cells (differentiated and nondifferentiated) that can be used for 

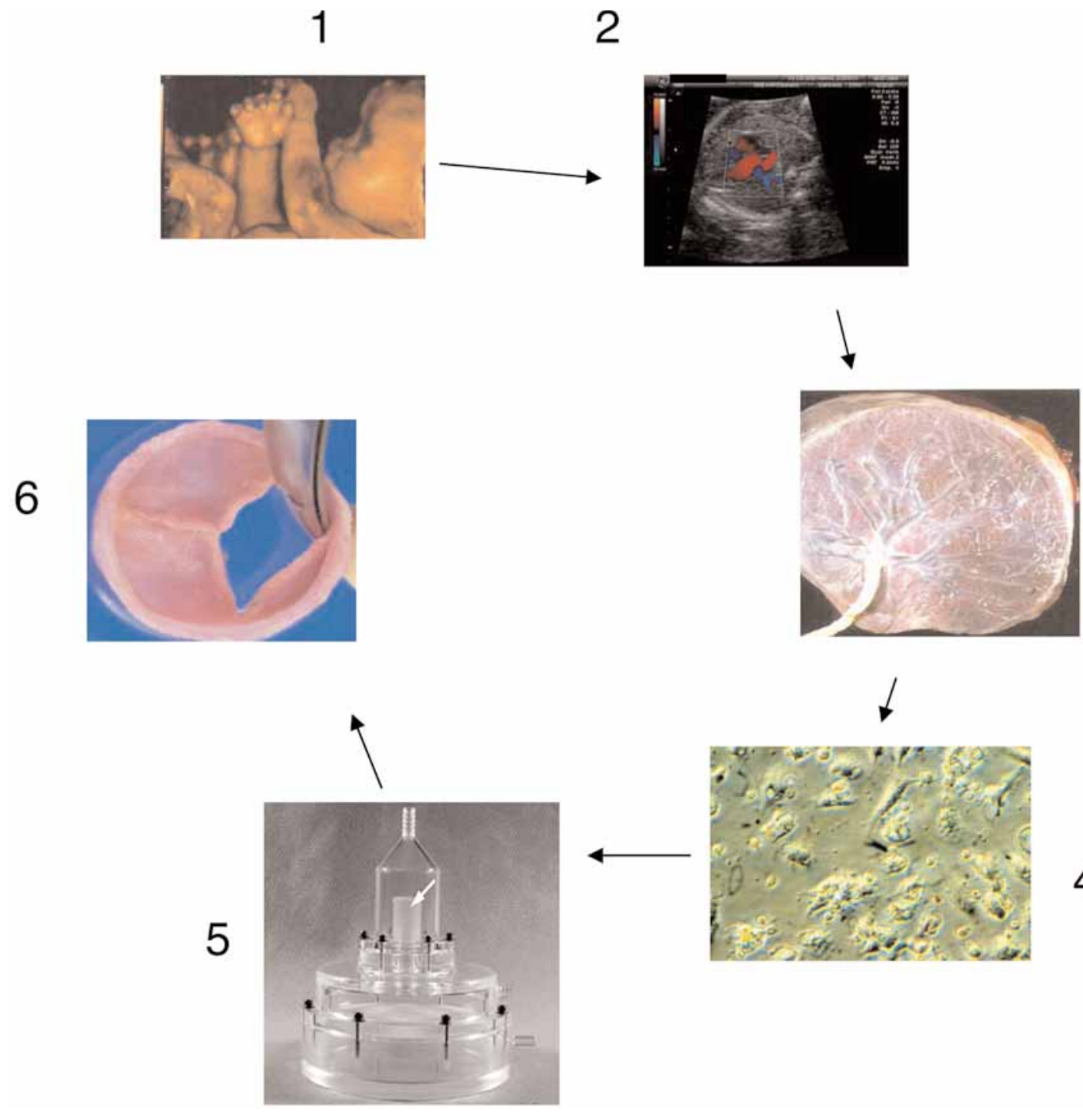

Fig. 2. Future perspective and scenario of using hUCC for cardiovascular tissue engineering: after prenatal diagnosis of the cardiovascular defect $(I, 2)$, umbilical cord blood is obtained (3). After isolation and differentiation (4), tissue-engineered constructs are positioned in a biomimetic system (5). After tissue maturation is sufficient, heart valves are ready for implantation.

Table 6

Challenges in Tissue Engineering

- Clinical and animal application trials

- Cost benefit ratio

- Cell sources (healthy, expandable cells)

- Optimization of scaffolds

- Scaled up bioreactors

- Prevention of tissue rejection

- Product preservation

cardiovascular tissue engineering as one example in regenerative medicine. There are numerous advantages of these autologous cells such as high immune tolerance, now major risk of transmitting bacterial or viral infections, capacity of growth and remodeling, less risk of thromboembolic complications and no sacrifice of intact donor structures.
A close cooperation between obstetric services (cell harvesting and acquisition) and the biotechnical laboratory (cell processing and tissue engineering) is needed to set up standards in quantity and quality control, sterility purposes, and sampling techniques. In addition various factors such as influence of type of birth (vaginal vs caesarean), vaginal contamination (meconium, bacteria, and so on), influence of maternal infections, and certainly fetal factors (age, weight, infection, gender, sex, and so on) have to be closely evaluated $(15,16)$.

At present the effect of crypreservation on the quality of hUCC as a source of tissue engineering compared with hUCC, which is immediately processed after birth is investigated. Further challenges are the clinical and animal application trials, investigation of future cell sources and their viability, optimization of scaffolds, scaled up bioreactors, prevention of tissue rejection, and product preservation (Table 6). 
If most of these questions will be answered, then there is a great opportunity and perspective to use hUCC for generating tissue-engineered vessels and heart valves for the repair of congenital malformations. In a future scenario after prenatal diagnosis, fetal cells would be immediately sampled and processed and the tissue engineered organ would be available after birth at the time of programmed heart surgery or some weeks later (Fig. 2).

\section{References}

1 Fauza D. Best Practice Clin Obstet Gynaecol 2004;18: 877-891.

2 Korbling M, Robinson S, Estrov Z. Cytotherapy 2005; 7(3):258-261.

3 Mayer J. Sem Thorac Cardiovasc Surg 1995;7(130-132).

4 Schoen FJ LR. J Biomed Mater Res 1999;47:439-465.

5 Hoerstrup SP, Sodian R, Sperling JS, et al. Tissue Eng 2000b; 6:75-79.
6 Zund G, Hoerstrup SP, Schoeberlein A, et al. Eur J Cardiothorac Surg 1998;13:160-164.

7 Shinoka T, Ma PX, Shum-Tim D, et al. Circulation 1996;94 (9 Suppl):164-168.

8 Shinoka T, Shum-Tim D, Ma PX, et al. J Thorac Cardiovasc Surg 1998;115:536-546.

9 Horstrup S, Kadner A, Breymann C. Ann Thor Surg 2002;74(1):46-52.

10 Kadner A, Hoerstrup S, Tracy J, et al. Ann Thorac Surg 2002;74:S1422-S1428.

11 Kadner A, Zund G, Maurus C, et al. Eur J Cardio-Thorac Surg 2004;25:635-641.

12 Schmidt D, Breymann C, Weber A, et al. Ann Thorac Surg 2004;78:2094-2098.

13 Schmidt D, Mol A, Neuenschwander S, et al. Eur J CardioThorac Surg 2005;27:795-800.

14 Schmidt D, Mol A, Breymann C, et al. Circulation 2006;114: 1125-1131.

15 Sanatloya J, Leon JD. JSoc Gynecological Invest 2005; 12(2):237A.

16 Solves P, Perales A, Moraga R. Acta Haematol 205;113(4):241-246. 